\title{
Digital Image Based Identification of Rice Variety Using Image Processing and Neural Network
}

\author{
Lilik Sumaryanti ${ }^{\star 1}$, Aina Musdholifah ${ }^{2}$, Sri Hartati ${ }^{2}$ \\ ${ }^{1}$ Department of Informatics, Engineering Faculty, Musamus University, Merauke, Indonesia \\ ${ }^{2}$ Department of Computer Science and Electronics, FMIPA UGM, Yogyakarta, Indonesia \\ *Corresponding author, e-mail: lilik.sumaryanti@gmail.com ${ }^{*}$, aina_m@ugm.ac.id ${ }^{2}$, shartati@ugm.ac.id ${ }^{3}$
}

\begin{abstract}
The consumer concern on the originality of rice variety and the quality of rice leads to originality certification of rice by existing institutions. Technology helps human to perform evaluations of food grains using images of objects. This study developed a system as a tool to identify rice varieties. Identification process was performed by analyzing rice images using image processing. The analyzed features for identification consist of six color features, four morphological features, and two texture features. Classifier used $L V Q$ neural network algorithm. Identification results using a combination of all features gave average accuracy of $70.3 \%$ with the highest classification accuracy level of $96.6 \%$ for Mentik Wangi and the lowest classification accuracy of $30 \%$ for Cilosari.
\end{abstract}

Keywords: rice, image processing, neural network, $L V Q$

Copyright $\odot 2015$ Institute of Advanced Engineering and Science. All rights reserved.

\section{Introduction}

Rice is one of the main food products for most world population, including Indonesians. The consumer concern on the originality of rice variety and the quality of rice leads to originality certification of rice by existing institutions. As a consequence, there should be an evaluation method for the originality of a product which is able to perform identification [1]. Identifying rice variety just by sight is generally very difficult because rice varieties are nearly identical visually, except for experts. In food industry, traditional evaluations are performed by several operators (trained instructors) using human sight. Along with technology development, it helps human to perform evaluations of food grains using images of objects [2, 3].

Morphological characteristics such as roundness, length, and density are widely used in assessing automatic sorting, detection and inspection of product quality in food industry [4]. Colors are one of the visual features which often used in image retrieval method [5, 6]. Texture feature analysis by machine vision technique classifies Iran grain types [7]. Grain type identification is based on morphological features and color features [8-10]. Image Analysis to classify grain types using color features and texture features uses neural network [11]. Classification of wheat grain varieties with input variables into the statistical model were the textures of single kernel projections, textures were computed separately for seven channels $(R$, $\mathrm{G}, \mathrm{B}, \mathrm{Y}, \mathrm{S}, \mathrm{U}, \mathrm{V})$ and the results were examined with the application of discriminant analysis and neural networks [12]. Classification of seed types uses MLP and neuro-fuzzy with color features, morphological features, and shape features [13]. The application of neural network to classify rice seed types is based on color features, morphological features and texture features $[14,15]$. Machine vision technique and neural network are used to identify corn seed types by combining mahalanobis distance discriminant and BPNN classifier [16]. Seed identification machine learning using combination of RBF and feed forward neural networks, weight training and bias algorithms use gradient descent with momentum and adaptive learning rate back propagation (BPPN) [17]. Probabilistic neural network (PNN) is used as a classifier for grading and quality testing of food grains [18].

This research developed a system to identify ten rice varieties based on digital images. Classification of rice varieties used LVQ neural network algorithm which consists of competitive layers which learned to classify the input vectors. If several input vectors were close to each other, those input vectors were categorized into the same class [19, 20]. 


\section{Materials and Methods}

Identification process using a neural network as a classifier that function to determines the class of each variety. The phases of activities that would perform in this study were shown in Figure 1, which consist of data collection (image acquisition), image analysis, feature extraction, and training by LVQ neural network algorithm. The creation of classification models by teaching network using case/pattern samples until managed to recognize patterns. Every time the output produced by the network didn't match the expected target, the weight would be renewed. The classification model contained optimal weights from the training stage which would be used during testing stage. If the identification results didn't match the expected targets, training stage would be performed again by changing neural network parameter values. The parameters were; learning rate, minimum error, and maximum epoch.

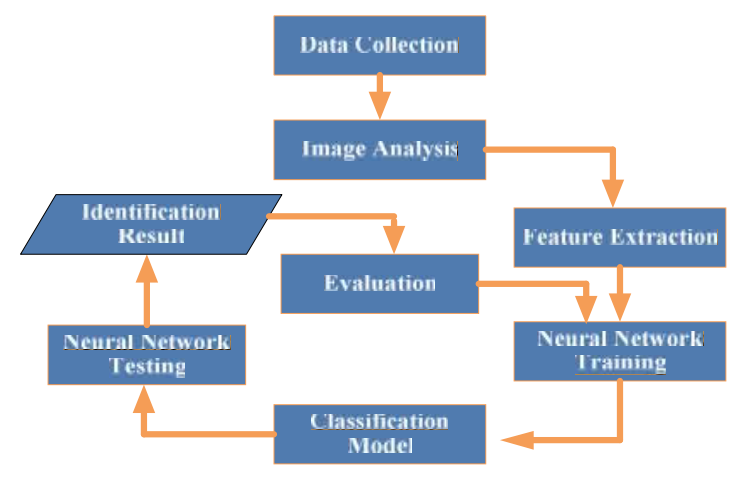

Figure 1. Research methodology

\subsection{Grain Sample}

The rice varieties used as samples in this study were ten rice varieties, which were Mentik Wangi, Pandan Wangi, Cilosari, Rajalele, Inpari 3, Inpari 6, IR66, IR64, Sintanur and Membramo from several different locations in Yogyakarta.

\subsection{Image Acquisition}

The camera used for image acquisition was Nikon Coolpix L810 with 16.1 megapixel resolution. For each variety, 100 images were taken, so total image of the research was 1000 images which were divided into two sets, 700 images for training data and 300 images for testing data. Figure 2 shows image acquisition method using a closed box to avoid any reflection and intervention of shadow caused by light. Lighting during image acquisition used a lamp in the box. To keep the lighting during image acquisition having the same level, a stabilizer was used to control electrical voltage. A hole in the middle of the top was provided for camera lens with fixed distance of $20 \mathrm{~cm}$ from rice grains.

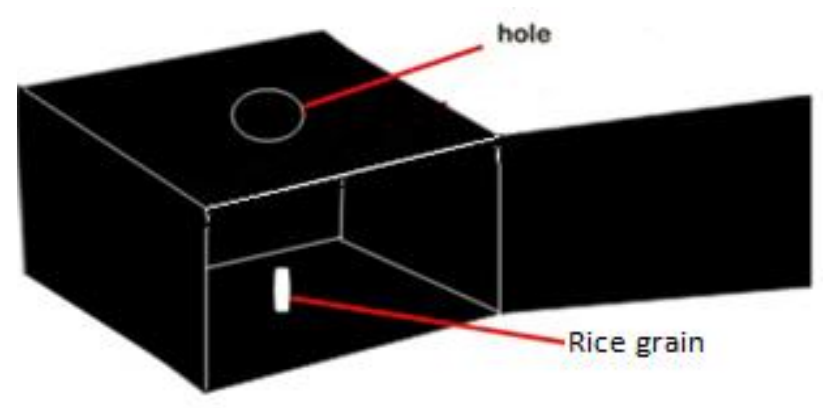

Figure 2. Image acquisition using box scheme 


\subsection{Design of Image Processing System}

Image processing and pattern recognition performed by analyzing the image of rice using a computer. The phases of activities for identification process were shown in Figure 3. The system design for image processing consisted of the following process components:

a) Load image was the process of retrieving image data stored in ".bmp" format in the same size, which was $300 \times 300$ pixel.

b) Preprocessing by conversing RGB images into grayscale images [21]. Grayscale image was converted into binary images by thresholding technique [22]. Internal contour tracing used Moore's algorithm [23].

c) Feature extraction measured quantitative information which was color, morphological, and texture features in rice grains to collect feature data.

d) Pattern recognition was the process of reintroducing patterns in the training and testing processes using data from feature extraction. Image Interpretation gave meanings to recognized objects to discover the success of data recognition.

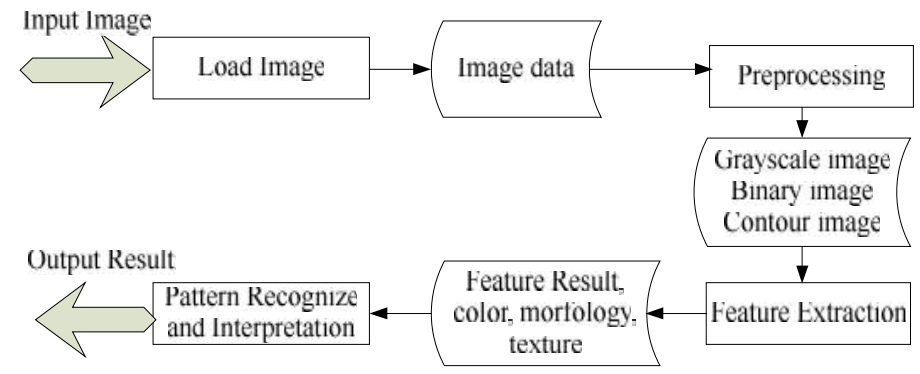

Figure 3. Block diagram of image processing

\subsection{Preprocessing}

RGB images illustrate color spaces using three basic components which red $(R)$, green $(G)$ and blue (B) in 3 bit spaces. Grayscale are images from conversions of RGB color images into images with small RGB color spaces in 1 bit spaces [24]. Conversion of RGB images into grayscale images used Formula (1) [21].

$$
\mathrm{Y}=\frac{\mathrm{R}+\mathrm{G}+\mathrm{B}}{3}
$$

Image segmentation is defined as the partitioning of an image into non overlapping, constituent regions that are homogenous with respect to some characteristic such as intensity or texture [25]. The technique used for image segmentation was thresholding segmentation technique $[2,8,13,14]$. The segmentation process would produce binary images, which are images with black and white grayness leveis. Generally, the development process of grayscale image to produce binary images used Formula (2) [22].

$$
g(x, y)=\left\{\begin{array}{l}
1 \text { jika } f(x, y) \geq T \\
0 \text { Jika } f(x, y)<T
\end{array}\right.
$$

Where $g(x, y)$ was binary image from grayscale image $f(x, y)$ and $T$ was threshold value. Edges of objects were discovered using contour following technique. The contour tracking used in this study was internal contour tracking which has been arranged by pixel location. The edges of objects were found using Moore's contour tracking algorithm [21, 23]. Figure 4 shows a sample of preprocessing result image. 


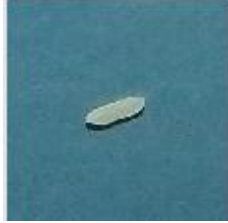

(a) RGB image



(b) grayscale image

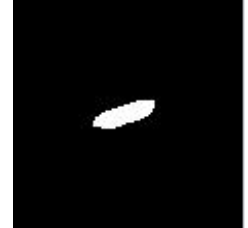

(c) segmented image

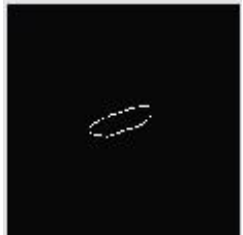

(d) Contour detection image

Figure 4. Sample of image resulted from preprocessing process

\subsection{Feature Extraction}

Process of computing the feature is known as feature extraction. Different features are chosen to describe different properties of the image [25]. The extracted features consist of 3 types which were color features, texture features, and morphological features. The six color features were; red mean, green mean, blue mean, hue mean, saturation mean and intensity mean $(R, G, B, H, S, I)$. The two texture features were taken from statistical calculation based on the histograms of grayscale images which were; mean and standard deviation, and the four morphological features were; area, perimeter, physiological length and physiological width.

\subsubsection{Color Features}

RGB color model based on the cartesian coordinate system, each color appears in the red, green, and blue primaries spectral components, the model is very satisfactory to the hardware's implementation, and matches the fact that human eye is sensitive to red, green, and blue, but do not reflect the essential differences among the colors, can not be consistent with human's perception of color. In order to easily describe the color image conducting color description, so as to in line with human's feeling facts of color based on the HSI color model [26]. Color features were calculated by averaging scores of every color component in RGB and HSI color spaces. The average provided measurements of color distribution and the calculation was applied on every R,G,B component using Formula (3) [21].

$$
=\frac{1}{M N} \sum_{i=1}^{M} \sum_{j=1}^{N} p_{i j}
$$

$\mathrm{M}$ and $\mathrm{N}$ were length and width values of pixels in image, and $\mathrm{P}_{\mathrm{ij}}$ was the color value in line $\mathrm{i}$ and column j.

$\mathrm{HSI}$ color space is a departure from the human visual system, which uses the Hue (Hue), color Saturation (Saturation), and Intensity (Intensity) to describe the color. HSI is to describe the color of a point inside the cylinder, the central axis of the cylindrical value since the black at the bottom to the top is white and among them is gray, angle around the axis corresponds to "hue", to this axis's distance corresponds to the "saturation", and the distance along this axis corresponds to the "brightness" [26]. Conversions of RGB color spaces into HSI used Formulas (4), (5), (6) and (7) [15].

$$
\begin{aligned}
& I=\frac{1}{3}(R+G+B) \\
& S=1-\frac{3}{(R+B+G)}[\min (R, G, B)] \\
& H=\left\{\begin{array}{c}
0, j i k a B \leq G \\
360-\theta, j i k a B>G
\end{array}\right. \\
& \theta=\cos ^{-1}\left\{\frac{[(R-G)+(R-B)] / 2}{\left[(R-G)^{2}+(R+B)(G-B)\right]^{1 / 2}}\right\}
\end{aligned}
$$

\subsubsection{Texture Features}

A simple method to get texture features was using histogram. Image histograms are diagram showing the frequency of every intensity value all over image pixel. Large values meant pixels had strong intensity. In grayscale images, total grayness level (denoted as L) was 256. 
Level values started from 0 to 255 . The first featured calculated statistically was average intensity using Formula (8) [21].

$$
\mathrm{m}=\sum_{\mathrm{i}=0}^{\mathrm{L}-1} \mathrm{i} . \mathrm{p}(\mathrm{i})
$$

i was grayness level in $f$ images and $p(i)$ was the probability of $i$, and $L$ was the highest grayness level value. The second feature was standard deviation which provided contrast value calculated by Formula (9) [21].

$$
\sigma=\sqrt{\sum_{\mathrm{i}=1}^{\mathrm{L}-1}(\mathrm{i}-\mathrm{m})^{2}} \mathrm{p}(\mathrm{i})
$$

\subsubsection{Morphological Features}

The morphological features of an object were determined by the edges of the objects [27]. Four morphological features were analyzed, which are area, perimeter, physiological length and physiological width. Area was evaluated by calculating pixels valued 255 in images. Perimeter of objects edges length obtained by calculating total pixel in the edges of rice grains. Physiological Length was the longest distance between two pixels in the edges of objects or known as diameter of objects [28]. Diameter could be calculated by "brute force" method with the algorithm shown in Figure 5 [21, 29].

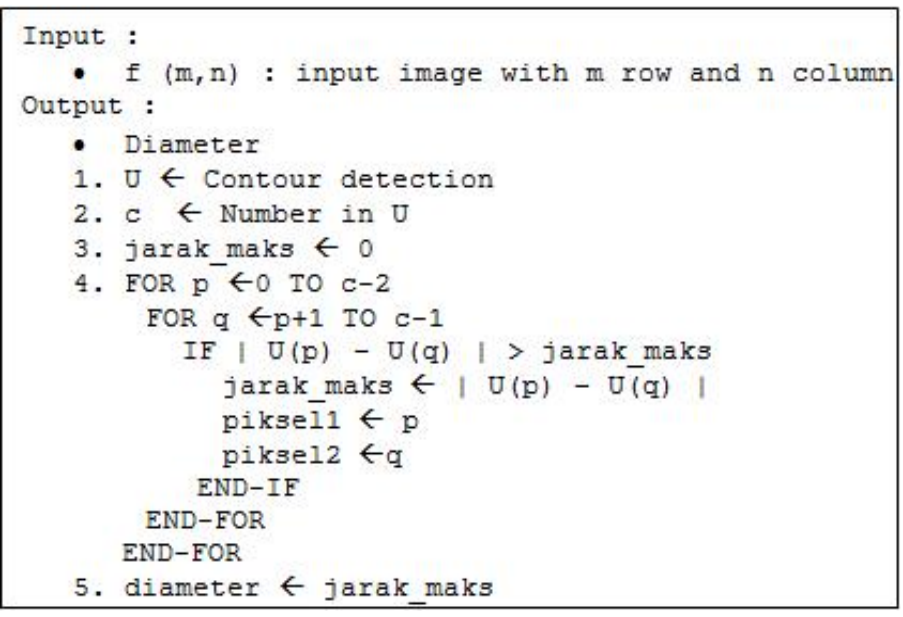

Figure 5. Algorithm of diameter estimation

Physiological Width was the longest line connecting two pixels in the edges of the objects which was perpendicular to maximum length of the objects [27]. After finding two points with the longest distance, gradient of the line going through both pixels was calculated using Formula (10) [21].

$$
\operatorname{grad} 1=\frac{y_{2}-y_{1}}{x_{2}-x_{1}}
$$

Lines perpendicular to gradient line which was grad1 had gradients of Formula (11) [21].

$$
\operatorname{grad} 2=-\frac{1}{\operatorname{grad} 1}
$$

\subsection{Identification of Varieties}

The identification process of rice varieties using LVQ neural network architecture consisted of input layers which have twelve neurons and output layers which have ten neurons. Extracted features which consisted of six color features, two texture features and four morphological features were used as input vectors in LVQ architecture. Each feature was represented by a neuron unit in an input layer, so the input layer had twelve neurons. An output 
layer consisted of 10 neurons which represent the number of classes for classification. Total neurons in output layers matched to number of rice varieties used in this study. LVQ neural algorithm trained on monitored competitive layers. Competitive layers learned to classify input vectors given. If several input vectors were close to each other, those input vectors were categorized into the same class [20].

Normalization or scaling process was performed on feature data used as input vectors of LVQ neural network, so that feature values were in certain intervals. Scaling process could be performed by Formula (12) [22].

$$
\hat{A}=\frac{A-A_{\min }}{A_{\max }-A_{\min }} *(S-R)+R
$$

Where $A$ is the original data, $\hat{A}$ the normalized input or output values, $A_{\max }$ and $A_{\min }$, are the maximum and minimum values of the concerned variable, respectively. $S$ and $R$ correspond to the desired values of the transformed variable range.

The creation of classification model by determining the expected parameter values, such as maximum epoch, learning rate, minimum error, learning rate decrement and initialization of initial weight values. Training process was performed to improve weight in the neural network by calculating input distance and pre-established initial weight initialization [20]. Neural network training was performed several times by changing parameter values to get the optimal parameter values for identification process.

\section{Results and Discussion}

Neural network training was performed by changing parameter values to get optimal parameters for identification process, i.e. learning rate using values from 0,001 to 1 , minimum error using values from 0.00001 to 0.00009 and maximum epoch parameter using values from 100 to 1000 . Figure 6 shows a graph of the result of neural network training with learning rate variations using maximum epoch of 1000 and minimum error of 0,00001 . Based on the graph, error of the smallest training occured at learning rate of 0.025 .

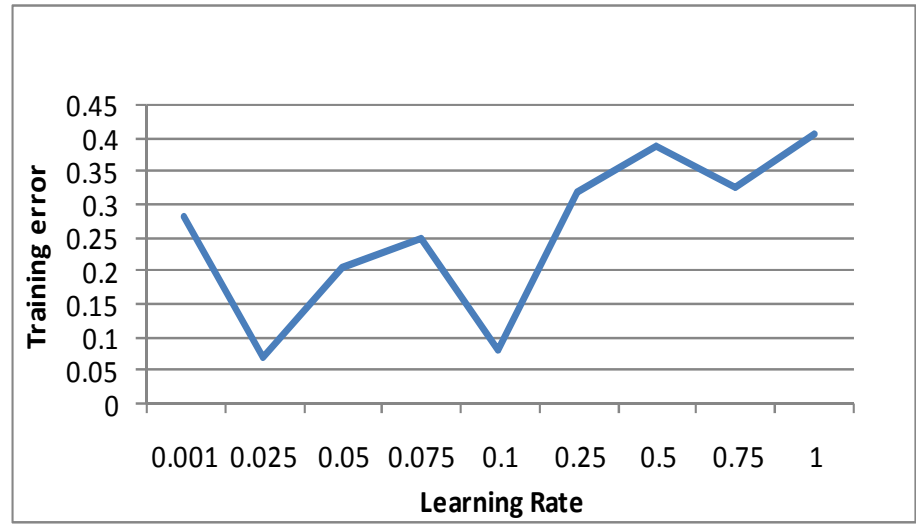

Figure 6. Training error with learning rate value variations

The trial to discover the result of neural network training was performed by changing the expected minimum error or target error parameter values. It used maximum epoch of 1000 and best learning rate value of 0,025 . Minimum error value used for training started from 0.00001 to 0.00009 . Training result showed that the smallest training error was 0.061428571 on Min $\alpha$ value of 0.00003 . Minimum error value of 0.00003 was then used in training trial by changing maximum epoch values from 100 to 1000. The training result by changing maximum parameters showed that iteration didn't impact the system because all results gave the same training error.

In this study, system testing used seven feature variations. The variations consist of color feature variation, texture feature variation, morphological feature variation, color feature 
and texture feature combination, color feature and morphological feature combination, morphological feature and texture feature combination and three feature combination from color feature, texture feature, and morphological feature. The accuracy of testing result using variation of combination all features with accuracy for each rice variety: $96,6 \%, 93,3 \%, 86,6 \%$, $70 \%, 63,3 \%, 50 \%, 80 \%, 30 \%$ for Mentik Wangi, Pandan Wangi, Rajalele, Sintanur, Membramo, IR64, IR66, Inpari 3, Inpari 6 and Cilosari, respectively. Cilosari has a low accuracy because of similarities in color and morphological features with other rice varieties.

Average accuracy of testing using feature variations show that variation of all features combination gave better accuracy than variation with one feature type or combination of two features type. Table 1 explains the test results using a combination of feature sets.

Table 1. Result test with feature variations

\begin{tabular}{clc}
\hline No & \multicolumn{1}{c}{ Feature variations } & Accuracy (\%) \\
\hline $\mathbf{1}$ & Combination of all feature types & 70.3 \\
$\mathbf{2}$ & Color features & 45 \\
$\mathbf{3}$ & Texture features & 37.6 \\
$\mathbf{4}$ & Morphological features & 50 \\
$\mathbf{5}$ & Combination of color and texture features & 39 \\
$\mathbf{6}$ & Combination of color and morphological features & 63.6 \\
$\mathbf{7}$ & Combination of texture and morphological features & 51 \\
\hline
\end{tabular}

An alternative approach to 'train and test' that is often adopted when the number of instances is small (and which many prefer to use regardless of size) is known as k-fold crossvalidation. If the dataset comprises $N$ instances, these are divided into $k$ equal parts, $k$ typically being a small number such as 4 . If $N$ is not exactly divisible by $k$, the final part will have fewer instances than the other $k-1$ parts. A series of $k$ runs is now carried out. Experiments carried out 4 times to measure the accuracy of the data. Each of the $k$ parts in turn is used as a test set and the other $k-1$ parts are used as a training set. The total number of instances correctly classified (in all $k$ runs combined) is divided by the total number of instances $N$ to give an overall level of predictive accuracy $p$. The average accuracy of the test results with a 4 -fold cross validation of $67.8 \%$.

The described experiment investigated 11 varieties of spring and winter wheat of different quality class. The analysis was performed on images acquired from a flatbed scanner interfaced to a PC. Kernel images were digitalized at high resolution (26734031) with 24-bit depth and $400 \mathrm{dpi}$. The variables input into the statistical model were the textures of single kernel projections. Textures were computed separately for seven channels (R, G, B, Y, S, U, V). The results wereexamined with the application of discriminant analysis and neural networks. The accuracy of texture-based classification of 11 wheat varieties reached $100 \%$. The experimental design which yielded the most satisfactory results comprised texture measurements from the combined area of 20 kernels and variables from seven channels input into the neural network [12]. Classification of 5 main rice grain varieties grown in different environments in Iran. Classification was made in terms of 24 color features, 11 morphological features and 4 shape factors that were extracted from color images of each grain of rice. The rice grains were then classified according to variety by multi layer perceptron (MLP) and neurofuzzy neural networks. The topological structure of the MLP model contained 39 neurons in the input layer, 5 neurons (Khazar, Gharib, Ghasrdashti, Gerdeh and Mohammadi) in the output layer and two hidden layers; neuro-fuzzy classifier applied the same structure in input and output layers with 60 rules. Average accuracy amounts for classification of rice grain varieties computed $99.46 \%$ and $99.73 \%$ by MLP and neuro-fuzzy classifiers alternatively [13]. The Presents neural networks approach for classification of 9 rice varieties. Algorithms were developed to extract thirteen morphological features, six colour features and fifteen texture features from colour images of individual seed samples. A different neural network models were developed for individual feature sets and for the combined feature set. High classification accuracy was given by textural features than morphological and colour features. An overall classification accuracy of $92 \%$ was obtained from combined feature model [14]. A digital image analysis algorithm based color and a morphological feature was developed to identify the six varieties (ey7954, syz3, xs11, xy5968, xy9308, z903) rice seeds which are widely planted in 
Zhejiang Province. Seven color and fourteen morphological features were used for discriminant analysis. Two hundred and forty kernels used as the training data set and sixty kernels as the test data set in the neural network used to identify rice seed varieties. When the model was tested on the test data set, the identification accuracies were $90.00 \%, 88.00 \%, 95.00 \%$, $82.00 \%, 74.00 \%, 80.00 \%$ for ey7954, syz3, xs11, xy5968, xy9308, z903 respectively [15].

\section{Conclusion}

The use of feature variations for testing showed that identification result using variation of all features combination gave better classification accuracy than variation of one feature type or variation of two feature type's combination. Testing result used variation of three feature types combination, i.e. color feature, texture feature, and morphological feature gave average accuracy of $70.3 \%$, with the highest classification accuracy of $96.6 \%$ for Mentik Wangi and the lowest classification accuracy $30 \%$ for Cilosari.

Future work should be arranged by combining aroma/scent features, using other features which wasn't used in this study, or applying different classification method to identify rice variety better.

\section{References}

[1] Khunkhett S, Remsungnen T. Non-Destructive Identification of Pure Breeding Rice Seed Using Digital Image Analysis. ${ }^{4}$ th IEEE Joint International Conference on Information and Communication Technology, Electronic and Electrical Engineering (JICTEE). 2014.

[2] Abirami S, Neelamegam P, Kala H. Analysis of Rice Granules using Image Processing and Neural Network Pattern Recognition Tool. International Journal of Computer Applications. 2014; 96(7): 09758887.

[3] Tahir AR, Neethirajan S, Jayas DS, Shahin MA, Symons SJ, White NDG. Evaluation of the Effect of Moisture Content on Cereal Grains by Digital Image Analysis. Science Direct Food Research International. 2007; 40: 1140-1145.

[4] Jayas DS, Paliwal J, Visen NS. Multi-layer Neural Networks for Image Analysis of Agricultural Products. J. Agric. Eng. Res. 2000; 77: 119-128.

[5] Golpour I, Jafar AP, Reza AC. Identification and Classification of Bulk Paddy, Brown, and White Rice Cultivars with Colour Features Extraction using Image Analysis and Neural Network. Czech J. Food Sci. 2014; 32(3): 280-287.

[6] Wang. Integrated Region Based Image Retrieval. Boston: Kluwer Academic Publisher. 2001.

[7] Pourreza A, Pourreza M, Abbaspour-Fard HM, Sadrnia H. Identification of Nine Iranian Wheat Seed Varieties by Textural Analysis with Image Processing. Jurnal Computers and Electronics in Agriculture. 2012; 83: 102-108.

[8] Pazoki A, Pazoki Z. Classification System for Rain Fed Wheat Grain Cultivars Using Artificial Neural Network, African Journal of Biotechnology. 2011; 10(41): 8031-8038.

[9] Mebatsion HK, Paliwal J, Jayas DS. Evaluation of Variations In The Shape of Grain Types Using Principal Components Analysis of the Elliptic Fourier Descriptors. Computers and Electronics in Agriculture. 2012; 80: 63-70.

[10] Mebatsion HK, Paliwal J, Jayas DS. Automatic Classification of Non Touching Cereal Grains in Digital Images Using Limited Morphological and Color Features. Computers and Electronics in Agriculture, 2013; 90: 99-105.

[11] Visen SN, Paliwal J, Jayas SD, White GDN. Image Analysis of Bulk Grain Samples Using Neural Networks. Jurnal Canadian Biosystems Engineering. 2004; 46.

[12] Zapotoczny P. Discrimination of Wheat Grain Varieties Using Image Analysis and Neural Networks. Journal of Cereal Science. 2011; 54: 60-68.

[13] Pazoki A, Farokhi F, Pazoki Z. 2014, Classification Of Rice Grain Varieties Using Two Artificial Neural Networks (MLP And Neuro-Fuzzy). Journal of Animal \& Plant Sciences. 2014; 24(1): 336-343.

[14] Silvia SC, Sonnadara U. Classification of Rice Grains Using Neural Networks. Proceedings of Technical Sessions. 2013; 29: 9-14.

[15] Zhao-yan L, Fang C, Yi-bin Y, Xiu-qin R. Identification of Rice Seed Varieties Using Neural Network. Journal of Zhejiang University SCIENCE. 2005; 6B(11): 1095-1100.

[16] Chen X, Xun Y, Wei L, Zhang J. Combining Discriminant Analysis and Neural Networks for Corn Variety Identification. Computers and Electronics in Agriculture, 2009; 71: 48-53.

[17] Anvarkhah S, Khajeh HM, Panah EDA. Seed Identification of Ten Rangeland Species Based on Machine Learning Using Combination of RBF and Feed Forward Neural Networks. Journal of Agriculture and Crop Sciences. 2012; 4(14): 993-1004. 
[18] Sidnal N, Patil VU, Patil P. Grading and Quality Testing of Food Grains Using Neural Network, International Journal of Research in Engineering and Technology (IJRET). 2013; 02.

[19] Utami E, Hartati S. Perancangan Perangkat Lunak untuk Pengenalan Karakter ASCII dari Gambar Bitmap Menggunakan Jaringan Syaraf Tiruan Metode Propagasi Balik. Jurnal Teknologi ACADEMIA ISTA. 2008; 2(2): 212-222.

[20] Kohonen T. Self-Organizing Maping. Third Edition. New York: Springer-Verlag Heidelberg. 2001.

[21] Kadir A, Susanto A. Teori dan Aplikasi Pengolahan Citra. Yogyakarta: Andi Offset. 2013.

[22] Putra D. Pengolahan Citra Digital. Yogyakarta: Andi Offset. 2010.

[23] Prada R, Kumar S, Agarwal R, Pradhan MP, Ghose MK. Contour Line Tracing Algorithm for Digital Topographics Maps. International Journal of Image Processing (IJIP). 2010. 4(2).

[24] Gonzales R, Woods RE. Digital Image Processing. Third Edition. Prentice-Hall: Eurson Education. 2008.

[25] Ehsani AR, Shafry MR, Norouzi A. Digital Dental X-Ray Image Segmentation and Feature Extraction. TELKOMNIKA. 2013; 11(6): 3109-3114.

[26] Yanhui X, Zhengyou W, Wan W, Jin W, Zheng W. Color Distortion of Digital Image and its Detection. TELKOMNIKA. 2013; 11(8): 4565-4571.

[27] Paliwal J, Visen NS, Jayas DS. Evaluation of Neural Network Architectures for Cereal Grain Classfication using Morphological Features. J.agric. Engng Res. 2001; 79(4): 361-370.

[28] Syahputra H, Harjoko A. Klasifikasi Varietas Tanaman Kelengkeng Berdasarkan Morfologi Daun Menggunakan Backpropagation Neural Network dan Probabilistic Neural Network. IJCCS. 2011; 5(3).

[29] Costa LF, Caesar RM. Shape Analysis and Classification Theory and Practice. Florida: CRC Press LLC. 2001. 